\title{
Assessing adhesive remnant removal and enamel damage with ultraviolet light: An in-vitro study
}

\author{
Alexandre Antonio Ribeiro, ${ }^{a}$ Layene Figueiredo Almeida, ${ }^{b}$ Lidia Parsekian Martins, ${ }^{c}$ \\ and Renato Parsekian Martins ${ }^{\mathrm{d}}$ \\ Natal, Rio Grande do Norte, and Araraquara, São Paulo, Brazil
}

\begin{abstract}
Introduction: Our objective was to assess the amounts of adhesive remnant and enamel damage when removing composite under conventional lighting and ultraviolet (UV) light. Methods: Thirty-eight premolars were divided into 2 groups according to the lighting used for adhesive removal. A multifluted tungsten-carbide bur at low speed was used for this process in both groups. The UV group received conventional lighting associated with UV light, and the conventional group received conventional lighting only. The amounts of adhesive remnant were calculated using images of the teeth taken under UV light with software. Scanning electron microscopy images of epoxy adhesive replicas of the teeth made before bonding and after adhesive removal were graded according to the enamel damage index (EDI), and the difference between the final and initial EDI scores was used for assessment of the enamel damage. To detect differences between the groups, the Mann-Whitney test was used to analyze the data from the adhesive remnant index and the EDI. Results: The conventional group had more adhesive left on the enamel $(P<0.001)$ than did the UV group. There was no difference between groups for the EDI $(P=0.729)$. Conclusions: UV lighting allows significantly more adhesive removal without increasing the damage caused to the enamel. (Am $\mathrm{J}$ Orthod Dentofacial Orthop 2017;151:292-6)
\end{abstract}

\section{A} $t$ the end of orthodontic treatment, the clinician needs to remove the adhesive left after debonding with as little damage as possible to the enamel. However, this extreme care during the process may prevent all adhesive from being removed; that in turn could cause 2 major problems. One is the possible creation of retentive areas that might favor biofilm buildup, subsequent decalcification, and carious lesions; the second problem involves

\footnotetext{
${ }^{a}$ Department of Orthodontics, Universidade Potiguar, Natal, Rio Grande do Norte, Brazil.

${ }^{\mathrm{b}}$ Program of Orthodontics, Universidade Estadual Paulista, Araraquara, São Paulo, Brazil.

${ }^{\mathrm{c}}$ Department and Program of Orthodontics, Universidade Estadual Paulista, Araraquara, São Paulo, Brazil.

dPrivate practice (Araraquara, São Paulo, Brazil); Program of Orthodontics, Universidade Estadual Paulista, Araraquara, São Paulo, Brazil.

All authors have completed and submitted the ICMJE Form for Disclosure of Potential Conflicts of Interest, and none were reported.

Address correspondence to: Renato Parsekian Martins, Rua Carlos Gomes, 2158, Araraquara, SP 14801340, Brazil; e-mail,dr_renatopmartins@hotmail.com.

Submitted, February 2016; revised and accepted, June 2016.

$0889-5406 / \$ 36.00$

(C) 2017 by the American Association of Orthodontists. All rights reserved.

http://dx.doi.org/10.1016/j.ajodo.2016.06.040
}

pigmentation of adhesive remnants caused by aging, leading to patient dissatisfaction. ${ }^{1,2}$

Even though the adhesive removal task appears simple and easy, excess bonding material is often overlooked because the orthodontic adhesive has a similar color to the enamel. Moreover, the use of filled adhesive sealants aimed at preventing white spot lesions further requires the material to blend in with the color of the enamel. ${ }^{3,4}$ The clinician must have confidence during adhesive removal that a clean, but damagefree enamel surface can be attained. However, to date, no damage-free adhesive removal technique has been described. ${ }^{5-7}$ Even though it is one of the safest methods for adhesive removal, ${ }^{8-10}$ the use of lowspeed multifluted tungsten-carbide burs will still cause injuries with depths varying from $0.05^{8}$ to $50 \mu \mathrm{m} .{ }^{11}$ Thus, it seems logical that an adhesive removal method that will not alter the adhesive color, but could render the removal process easier and possibly safer would be advantageous during the debonding process.

More recently, ultraviolet light (UV) fluorescent chemicals have been added to orthodontic adhesives, allowing UV light to be used as an aid to adhesive remnant removal. In addition, light-curing devices 
with UV light filters are already available on the market, allowing this alternative method to be clinically applied. $^{12,13}$ Nevertheless, the use of this technology has not been tested scientifically; therefore, its effectiveness remains questionable.

Based on the potential of causing the least damage to dental enamel and the lack of orthodontic studies on the subject, the aim of this study was to assess whether the removal of adhesive with fluorescent properties under UV light is more effective and causes less damage to enamel than conventional lighting.

\section{MATERIAL AND METHODS}

Due to a lack of similar studies in the literature and because the brackets were removed by a shear bond strength test, the sample size calculation was carried out with data from the literature on shear bond strength tests. A sample size of 18 teeth was found to be adequate using data from the literature. ${ }^{12}$ The number was determined with significance of 5\% and power of $80 \%$; moreover, to ensure that an appropriate sample was available if any unavoidable failure occurred, 20 specimens were used per group.

A total of 40 premolars with no brackets previously bonded and free of caries, fractures, and restorations were acquired from the tooth bank of the Faculdade de Odontologia de Araraquara (Universidade Estadual Paulista). The use of these teeth for this research was approved by the ethics and research project committee of the university, collected through donations, with consent from the patients, and after extractions for orthodontic or periodontal reasons. The teeth were stored in distilled water at $4^{\circ} \mathrm{C}$ for not longer than 3 months according to the International Organization for Standardization standard 11405:2014.

The teeth were vertically embedded in acrylic resin in polyvinyl chloride cylinders (diameter, 0.5 in; height, $3 \mathrm{~cm})$ up to the cementoenamel junction. A metal square was used to align the surfaces of the teeth perpendicular to the bottom of the cylinder.

The surfaces of the premolars were cleaned and pumiced with an oil-free paste and a rubber cup for $15 \mathrm{sec}-$ onds, washed, and completely air dried for 10 seconds with oil-free air and water. The buccal dental enamel was conditioned with 37\% phosphoric acid for $30 \mathrm{sec}-$ onds, washed, and completely dried for 10 seconds with oil-free air and water. The liquid adhesive was applied (Opal Seal; Opal Orthodontics, South Jordan, Utah) and light cured for 6 seconds with the VALO LED (Ultradent, South Jordan, Utah) on extra power. All premolars had 0.022-in slot brackets (Opal Orthodontics) bonded to them using the UV-sensitive composite Opal Bond MV
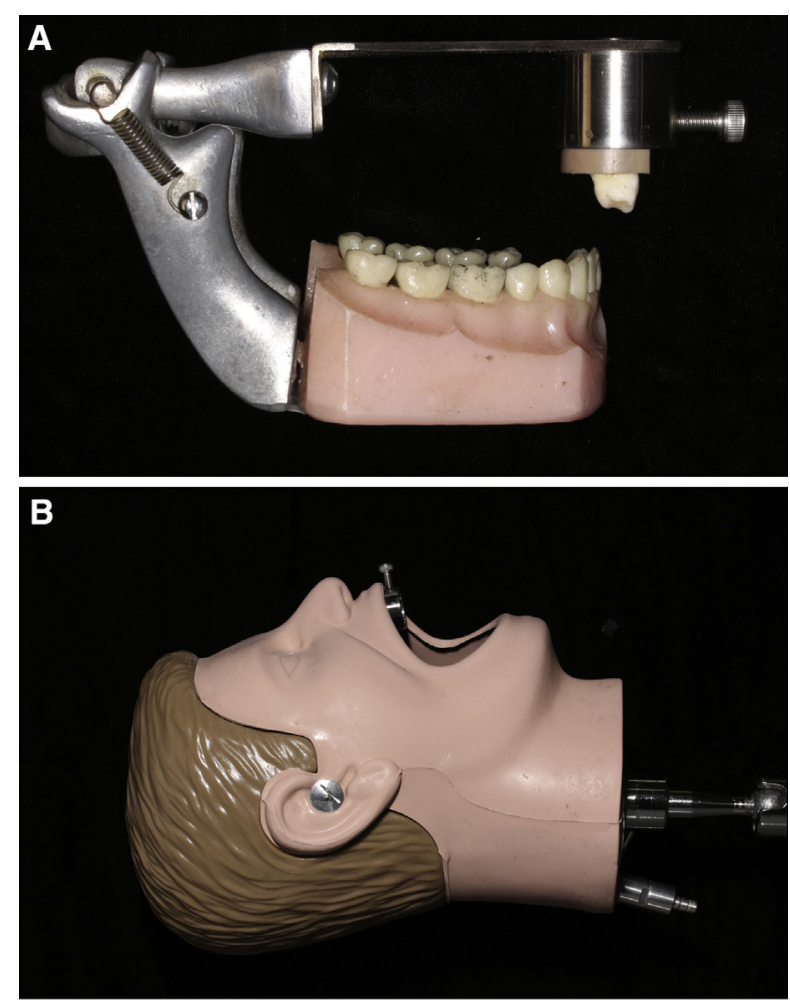

Fig 1. A, Mannequin with a specimen attached; B, mannequin head with the specimen attached for adhesive removal.

(Opal Orthodontics). The brackets used had a bonding base with an average area of $11.045 \mathrm{~mm}^{2}$.

The specimens were maintained in distilled water at $37^{\circ} \mathrm{C}$ for 24 hours before the shear bond strength test, which showed adequate bond strength, ranging from 13.16 to $19.43 \mathrm{MPa}$. Since 1 tooth was fractured during the test, another was randomly removed from the sample to produce 2 equal groups.

After debonding, the teeth were randomly divided into 2 groups of 19 according to the lighting to be used for removal of the adhesive remnants. The adhesive removal of 1 group was done under conventional lighting emitted by a dental chair unit, and the operator (L.F.A.) used a UV light-emitting diode flashlight to reveal the adhesive by fluorescence, whereas the other group received conventional lighting only. The procedure was performed with a 12-bladed tungsten-carbide bur (Orthometric, Marília, São Paulo, Brazil) in a lowspeed hand piece. To make the procedure similar to that performed clinically, the teeth were adapted to a mannequin head for the removal procedure (Fig 1).

To assess the amount of adhesive remnant on the enamel surface, the teeth were photographed under a 


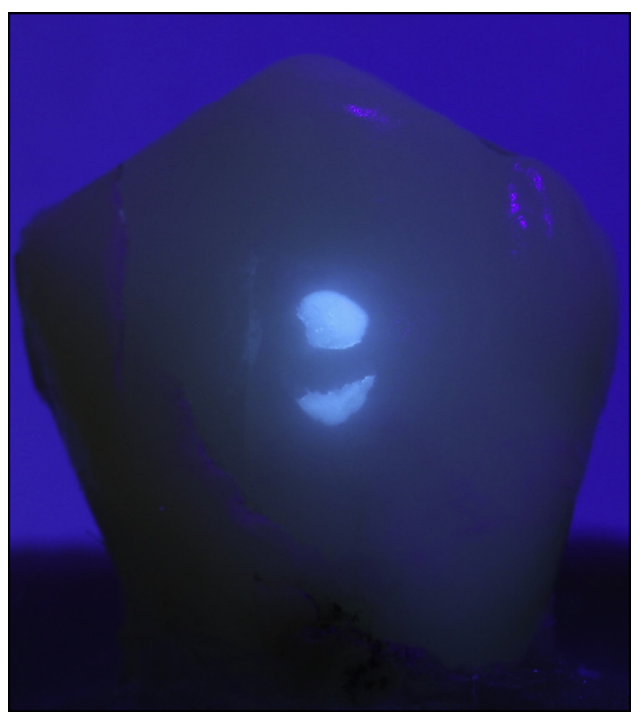

Fig 2. A specimen under UV light used for measurement of the area of adhesive remnants.

UV light filter adapted to a light-emitting diode (VALO, Ultradent) (Fig 2) using a digital camera (EOS Rebel T3i; Canon, Tokyo, Japan) with a 90-mm macro lens, 1/60 shutter speed, F 8.0, and ISO 400. The photographs were scaled, and the area of adhesive remaining after the removal process was measured by lmage $\mathrm{J}$ software (version 1.36; National Institutes of Health, Rockville, Md). Two measurements were taken by the same operator (L.F.A.) a week apart to evaluate the method error using Bland-Altman plots. Bias was 0.04, and the limits of agreement ranged from 0.38 to $-0.46 \mathrm{~mm}^{2}$ (Fig 3). To reduce error, the average of the 2 measurements of each specimen was for the statistical analyses.

Enamel damage was assessed using epoxy resin replicas (Epofix; Struers, Ballerup, Denmark), which were made by taking impressions of the specimens before bonding and after adhesive removal with light polyvinylsiloxane material (Zhermack, Badia Polesine, Italy).

The replicas were coated with gold and photographed by scanning electron microscopy (JEOL, Tokyo, Japan) under 20-times magnification. One calibrated and blinded operator (L.F.A.) graded the enamel damage of all the specimens before bonding and after adhesive removal in a random sequence and assigning each photograph a score according to the Enamel Damage Index (EDI; Table 1). ${ }^{13}$ The after-adhesive-removal score (Fig 4, A) was subtracted from the before-bonding score (Fig 4, B) for comparison of the groups. Intraoperator error was determined by repeating measurements after 7 days and analyzing these by a weighted kappa test; this resulted in a value of 0.9 .

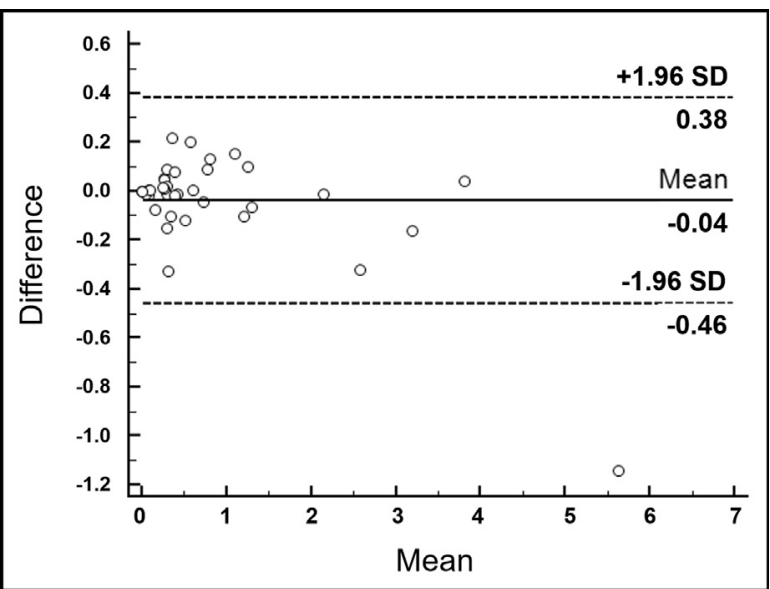

Fig 3. Bland-Altman plot of the difference between repeated measurements; the continuous line depicts the average, and the dotted lines depict the standard deviations.

\section{Table I. EDI scores and characteristics}

$\begin{array}{ll}\text { Score } & \text { Surface characteristics } \\ 0 & \text { Smooth surface without scratches, visible perikymata* } \\ 1 & \text { Acceptable surface with fine scratches spread } \\ 2 & \begin{array}{c}\text { Rough surface with several rough scratches or visible } \\ \text { minor grooves }\end{array} \\ 3 & \begin{array}{c}\text { Surface with rough scratches, large grooves, and enamel } \\ \text { damage visible with the naked eye }\end{array}\end{array}$

*Perikymata are transverse wavy ridges that correspond to the incremental lines of Retzius.

SPSS software (version 20; 1BM, Armonk, NY) was used for statistical testing. The Mann-Whitney test with a 95\% significance level $(P<0.05)$ was used to detect differences between groups 1 and 2 according to the adhesive remnant index and the EDI.

\section{RESULTS}

There was a significant difference in the adhesive remnant scores between the groups $(P<0.001)$. The UV light group had a median of $0.25 \mathrm{~mm}^{2}$ of adhesive remnant, and the conventional group had a median of $0.80 \mathrm{~mm}^{2}$ (Table 11$)$.

There was no difference in enamel damage between the UV and conventional lighting groups $(P=0.703)$. In the UV group, in $36.8 \%$ of the specimens, the EDI increased by 1 point, in $36.8 \%$ it increased by 2 points, and in $10.5 \%$ of the group it increased 3 points (Table $111)$. In the conventional lighting group, $15.8 \%$ of specimens had a 1-point increase in the EDl, $21.1 \%$ had a 2-point increase, and 10\% had a 3-point increase. 

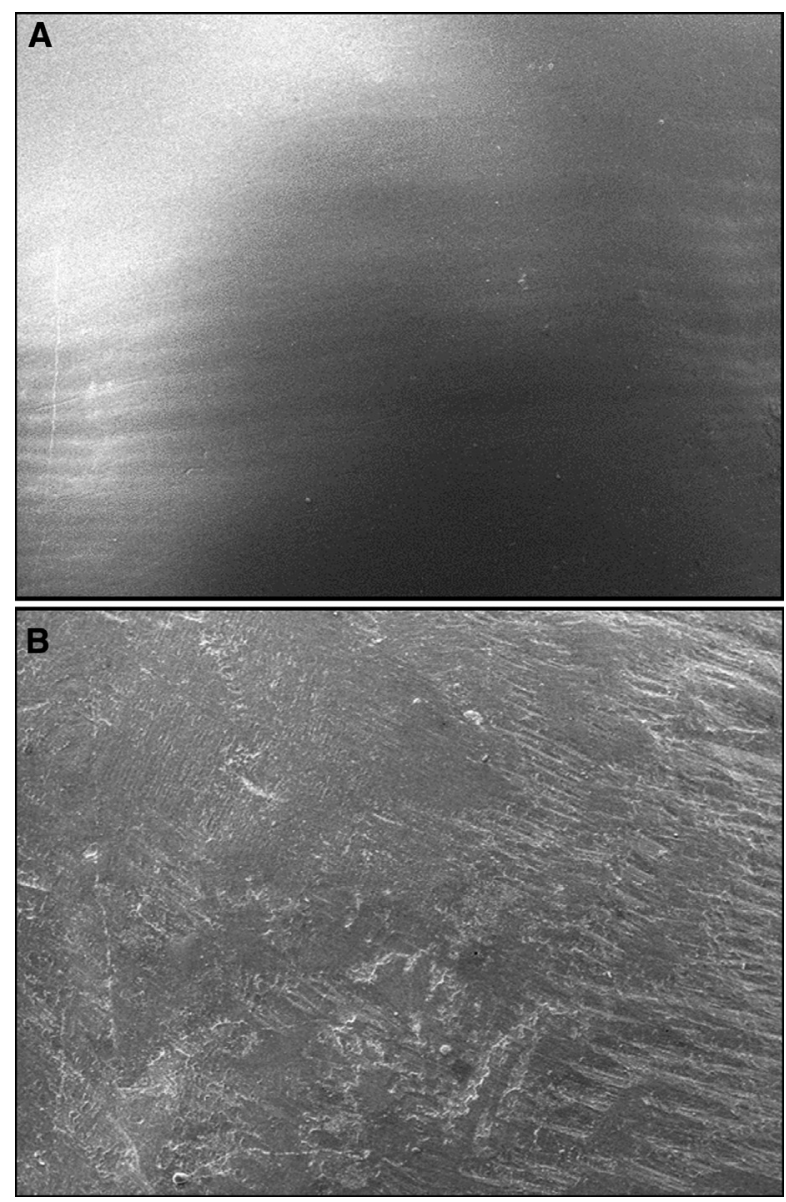

Fig 4. A, Scanning electron microscope photograph under 20-times magnification of a specimen before bonding with an EDI score of 0 ; $\mathbf{B}$, scanning electrom microscope photograph under 20-times magnification of a specimen after removal with an EDI score of 2 .

Table II. Results of Mann-Whitney test comparing the adhesive remnants on the enamel surfaces in both groups $(P<0.05)$

\begin{tabular}{lcccc} 
& \multicolumn{3}{c}{ Percentile $\left(\mathrm{mm}^{2}\right)$} & Mann-Whitney \\
\cline { 2 - 4 } Variable & 25 & 50 (median) & 75 & $(\mathrm{P}<0.05)$ \\
UV light & 0.05 & 0.25 & 0.35 & $(P<0.001)$ \\
$\begin{array}{c}\text { Conventional } \\
\text { lighting }\end{array}$ & 0.34 & 0.80 & 2.14 &
\end{tabular}

\section{DISCUSSION}

More adhesive was removed from the enamel surface when the UV light was used to assist the procedure. Both liquid and paste adhesives used in the study had fluorescent properties, which allowed them to be easily identified and effectively removed. This outcome
Table III. Distribution of EDI differences (T2-T1) for the groups

\begin{tabular}{|c|c|c|c|c|c|c|c|c|c|}
\hline \multirow[b]{2}{*}{ Group } & \multirow{2}{*}{$\frac{\text { Total }}{n}$} & \multicolumn{2}{|c|}{ EDI 0} & \multicolumn{2}{|c|}{ EDI 1} & \multicolumn{2}{|c|}{ EDI 2} & \multicolumn{2}{|c|}{ EDI 3} \\
\hline & & $n$ & $\%$ & $n$ & $\%$ & $n$ & $\%$ & $n$ & $\%$ \\
\hline UV light & 19 & 3 & 15.8 & 7 & 36.8 & 7 & 36.8 & 2 & 10.5 \\
\hline $\begin{array}{c}\text { Conventional } \\
\text { lighting }\end{array}$ & 19 & 3 & 15.8 & 4 & 21.1 & 12 & 63.2 & 0 & 0 \\
\hline
\end{tabular}

adds importance to 2 other studies suggesting that restorative composite can be identified through fluorescence to differentiate it from the dental structure for ease at removal. ${ }^{14,15}$ Nevertheless, both studies tested a few restorative adhesives available on the market to identifying which one had fluorescent properties under several light wavelengths. Those authors, however, did not test composite and adhesive removal, as we did in this study. Interestingly, fluorescence might be used not only to reveal the adhesive exposed to an invisible light wavelength, as in our study, but also to disguise its presence when it is exposed to a visible light wavelength. $^{16,17}$

The use of UV light to assist the removal of adhesive with multiblade tungsten-carbide burs did not cause more damage than did conventional lighting. It was expected that removal under UV light illumination would cause less damage to enamel, since it allows a clear distinction of the tooth surface from the adhesive. There are no other studies in the literature answering why that did not happen. Our results might be explained by the instrument used for adhesive removal-a multibladed bur-not allowing the process to be damage-free, ${ }^{10}$ or perhaps adhesive removal might cause proportional damage, with more damage to enamel as more adhesive is removed.

In this study, no polishing was carried out to prevent confounding of the results, since the enamel damage could have been disguised. In addition, this would have added another source of variation to the research measurements.

Among the methods to remove orthodontics adhesives, the addition of UV-sensitive materials to the adhesive associated with UV lighting at removal appears to be a safe and practical method. The other methods already proposed in the literature are quantitative light-induced fluorescence, ${ }^{18}$ which uses an expensive device, and is hard to be used clinically on a day-to-day basis because it requires a dark environment; brilliant green or ink dye, ${ }^{19}$ which is not approved by the Food and Drug Administration and is toxic; articulating paper ${ }^{20}$; food coloring $^{21}$ techniques, both of which might not be practical, since the surface of the composite needs to be 
revealed again after each small layer is removed; and thermo-photochromatic adhesives (Greengloo and Bluegloo; Ormco, Glendora, Calif), which could very well be used for evincing composite flash but would probably cause esthetic issues during treatment. Since these composites undergo a color change at temperatures just below body temperature, they can become visible when patients eat or drink cold food or beverages. Even though these composites have been shown to produce adequate bond strengths ${ }^{22-24}$ and similar microleakage under brackets compared with conventional composites, ${ }^{25}$ they have not been tested for their effectiveness during removal.

The goal of this project was to simulate the clinical situation as closely as possible; however, it is still difficult to extrapolate the results directly from the in-vitro to the in-vivo situations. These results provide clinically useful information, but we emphasize that more clinical studies are needed to confirm the results.

\section{CONCLUSIONS}

The use of UV light associated with a fluorescent adhesive allows for efficient adhesive removal compared with conventional lighting, without causing additional damage to the enamel.

\section{REFERENCES}

1. Eliades T, Gioka C, Heim M, Eliades G, Makou M. Color stability of orthodontic adhesive resins. Angle Orthod 2004;74:391-3.

2. Karamouzos A, Athanasiou AE, Papadopoulos MA, Kolokithas G. Tooth-color assessment after orthodontic treatment: a prospective clinical trial. Am J Orthod Dentofacial Orthop 2010;138:537.e1-8: discussion, 537-9.

3. Hu W, Featherstone JD. Prevention of enamel demineralization: an in-vitro study using light-cured filled sealant. Am J Orthod Dentofacial Orthop 2005;128:592-600.

4. Benham AW, Campbell PM, Buschang PH. Effectiveness of pit and fissure sealants in reducing white spot lesions during orthodontic treatment. A pilot study. Angle Orthod 2009;79:338-45.

5. Zachrisson BU, Arthun J. Enamel surface appearance after various debonding techniques. Am J Orthod 1979;75:121-7.

6. Pont HB, Ozcan M, Bagis B, Ren Y. Loss of surface enamel after bracket debonding: an in-vivo and ex-vivo evaluation. Am J Orthod Dentofacial Orthop 2010;138:387.e1-9: discussion, 387-9.

7. Ozer T, Basaran G, Kama JD. Surface roughness of the restored enamel after orthodontic treatment. Am J Orthod Dentofacial Orthop 2010;137:368-74.
8. Hosein 1, Sherriff M, lreland AJ. Enamel loss during bonding, debonding, and cleanup with use of a self-etching primer. Am J Orthod Dentofacial Orthop 2004;126:717-24.

9. Ireland AJ, Hosein 1, Sherriff M. Enamel loss at bond-up, debond and clean-up following the use of a conventional light-cured composite and a resin-modified glass polyalkenoate cement. Eur J Orthod 2005;27:413-9.

10. Ogaard B, Fjeld M. The enamel surface and bonding in orthodontics. Semin Orthod 2010;16:37-48.

11. Al Shamsi AH, Cunningham JL, Lamey PJ, Lynch E. Three-dimensional measurement of residual adhesive and enamel loss on teeth after debonding of orthodontic brackets: an in-vitro study. Am J Orthod Dentofacial Orthop 2007;131:301.e9-15.

12. Carvalho PE, dos Santos VM, lsber H, Cotrim-Ferreira FA. Halogen light versus LED for bracket bonding: shear bond strength. Dental Press J Orthod 2013;18:31.e1-6.

13. Schuler FS, van Waes H. SEM-evaluation of enamel surfaces after removal of fixed orthodontic appliances. Am J Dent 2003;16: 390-4.

14. Tani K, Watari F, Uo M, Morita M. Discrimination between composite resin and teeth using fluorescence properties. Dent Mater J 2003;22:569-80.

15. Bush MA, Hermanson AS, Yetto RJ, Wieczkowski G Jr. The use of ultraviolet LED illumination for composite resin removal: an in vitro study. Gen Dent 2010;58:e214-8.

16. Panzeri H, Fernandes LT, Minelli CJ. Spectral fluorescence of direct anterior restorative materials. Aust Dent J 1977;22:458-61.

17. Lee YK, Lu H, Powers JM. Fluorescence of layered resin composites. J Esthetic Restor Dent 2005;17:93-100.

18. Pretty $1 A$, Hall AF, Smith PW, Edgar WM, Higham SM. The intraand inter-examiner reliability of quantitative light-induced fluorescence (QLF) analyses. Br Dent J 2002;193:105-9.

19. Abdallah MN, Light N, Amin WM, Retrouvey JM, Cerruti M, Tamimi F. Development of a composite resin disclosing agent based on the understanding of tooth staining mechanisms. J Dent 2014:42:697-708.

20. Rachala MR, Kishore MS, Vamsilatha K. Staining adhesive remnants for easy removal. J Clin Orthod 2013;47:672.

21. Campbell PM. Enamel surfaces after orthodontic bracket debonding. Angle Orthod 1995;65:103-10.

22. lzadi Ml, Sherriff M, Cobourne MT. A comparative investigation into relative bond strengths of Damon3, Damon3MX, and APC 11 brackets using different primer and adhesive combinations. Eur J Orthod 2012;34:778-82.

23. Reicheneder CA, Gedrange T, Lange A, Baumert U, Proff P. Shear and tensile bond strength comparison of various contemporary orthodontic adhesive systems: an in-vitro study. Am J Orthod Dentofacial Orthop 2009;135:422.e1-6: discussion, 422-3.

24. Lowder PD, Foley T, Banting DW. Bond strength of 4 orthodontic adhesives used with a caries-protective resin sealant. Am J Orthod Dentofacial Orthop 2008;134:291-5.

25. Alkis H, Turkkahraman H, Adanir N. Microleakage under orthodontic brackets bonded with different adhesive systems. Eur J Dent 2015;9:117-21. 